\title{
A REMARK ON BOUNDEDNESS OF THE NUMERICAL RANGE
}

\author{
CHI FAI CHAN
}

\begin{abstract}
It is shown that Chien's result on the smallest rectangle with sides parallel to the axes containing the numerical range of a real matrix follows from a simple observation on complex matrices. A counter-example to Chien's conjecture is also given.
\end{abstract}

The numerical range of an $m$-square complex matrix $B$ is defined to be

$$
W(B)=\left\{(B x, x):\|x\|=1, \quad x \in \mathbb{C}^{m}\right\},
$$

where (.,.) denotes the standard inner product in $C^{m}$. Let $\Lambda(B)$ denote the set of eigenvalues of $B$. If $B$ is hermitian, let

$$
\lambda_{1}(B)=\max \Lambda(B) \quad \text { and } \quad \lambda_{m}(B)=\min \Lambda(B) .
$$

Let $A=\left[a_{i j}\right]$ be an $n$-square complex matrix. Then, for $x \in \mathcal{C}^{n}$,

$$
(A x, x)=\frac{1}{2}\left(\left(A+A^{*}\right) x, x\right)+\frac{i}{2}\left(i\left(A^{*}-A\right) x, x\right) .
$$

Since $A+A^{*}$ and $i\left(A^{*}-A\right)$ are hermitian, it follows that

$$
W\left(A+A^{*}\right)=\left[\lambda_{n}\left(A+A^{*}\right), \lambda_{1}\left(A+A^{*}\right)\right],
$$

and

$$
W\left(i\left(A^{*}-A\right)\right)=\left[\lambda_{n}\left(i\left(A^{*}-A\right), \lambda_{1}\left(i\left(A^{*}-A\right)\right)\right] .\right.
$$

Hence $[a, b] \times[c, d]$ is the smallest rectangle with sides parallel to the axes containing $W(A)$, where

$$
\begin{aligned}
& a=\frac{1}{2} \lambda_{n}\left(A+A^{*}\right), \\
& b=\frac{1}{2} \lambda_{1}\left(A+A^{*}\right), \\
& c=\frac{1}{2} \lambda_{n}\left(i\left(A^{*}-A\right)\right)=\frac{1}{2} \min \left\{\operatorname{Im}(\lambda): \lambda \in \Lambda\left(A-A^{*}\right)\right\}, \\
& \text { and } \\
& d=\frac{1}{2} \lambda_{1}\left(i\left(A^{*}-A\right)\right)=\frac{1}{2} \max \left\{\operatorname{Im}(\lambda): \lambda \in \Lambda\left(A-A^{*}\right)\right\} .
\end{aligned}
$$

Received October 12, 1990. 
Suppose now that $A$ is real. M. T. Chien [1] proved that

$$
c=\frac{1}{2} \lambda_{n}(K) \quad \text { and } \quad d=\frac{1}{2} \lambda_{1}(K),
$$

where

$$
K=\left[\begin{array}{cc}
0 & A-A^{T} \\
A^{T}-A & 0
\end{array}\right] .
$$

We shall see that a shorter proof is obtained from the previous observation. It is obvious (see, e.g., [2, pp. 45, 46]) that

$$
\Lambda\left(\left[\begin{array}{cc}
0 & B \\
B^{*} & 0
\end{array}\right]\right)=\left\{\alpha \in \mathbb{R}: \alpha^{2} \in \Lambda\left(B B^{*}\right)\right\}
$$

As $A-A^{T}$ is skew-symmetric,

$$
\begin{aligned}
\Lambda(K) & =\left\{\alpha \in \mathbb{R}: \alpha= \pm \operatorname{Im}(\lambda), \quad \lambda \in \Lambda\left(A-A^{T}\right)\right\} \\
& =\left\{\alpha \in \mathbb{R}: \alpha=\operatorname{Im}(\lambda), \quad \lambda \in \Lambda\left(A-A^{T}\right)\right\},
\end{aligned}
$$

and Chien's result follows. Note also that $\lambda_{n}(K)=-\lambda_{1}(K)$, so $c=-d$.

We study some particular cases:

(i) $n=2$. $\Lambda\left(A-A^{T}\right)=\{\lambda i,-\lambda i\}$, where $\lambda \geq 0$, and the eigenvalues of $K$ are $\pm \lambda$.

Since

$$
\begin{aligned}
2 \lambda^{2} & =\operatorname{tr}\left(\left(A-A^{T}\right)^{T}\left(A-A^{T}\right)\right)=\sum_{i, j}\left(a_{i j}-a_{j j}\right)^{2}, \\
\lambda^{2} & =\sum_{i<j}\left(a_{i j}-a_{j i}\right)^{2} .
\end{aligned}
$$

(ii) $n=3$. $\Lambda\left(A-A^{T}\right)=\{\lambda i,-\lambda i, 0\}$, where $\lambda \geq 0$, and the eigenvalues of $K$ are $0, \pm \lambda$. Similarly, we have (1).

Chien [1] conjectured for any $n$ that if $\lambda$ is an eigenvalue of $K$, then $\lambda=0$ or $\lambda$ satisfies (1).

(iii) $n=4$. $\Lambda\left(A-A^{T}\right)=\{\lambda i,-\lambda i, \mu i,-\mu i\}$, where $\lambda, \mu \geq 0$, and the eigenvalues of $K$ are $\pm \lambda, \pm \mu$. Similarly,

$$
\lambda^{2}+\mu^{2}=\sum_{i<j}\left(a_{i j}-a_{j i}\right)^{2} .
$$

Let $B=\left[\begin{array}{ll}0 & 1 \\ 0 & 0\end{array}\right]$. Then $\Lambda\left(B-B^{T}\right)=\{i,-i\}$. Let $A=B \oplus B$. Then the eigenvalues of $K$ are \pm 1 , whereas $\sum_{i<j}\left(a_{i j}-a_{j i}\right)^{2}=2$. Chien's conjecture fails.

\section{Acknowledgement}

The author is grateful to Dr. Yik-IIoi Au-Yeung for his guidance and encouragement and to Mr. Che-Man Cheng for his useful comment. 


\section{References}

[1] M. T. Chien, "Boundedness of the numerical range", Linear Algebra Appl.134, 25-30, 1990.

[2] F. R. Gantmacher, The. Theory of Matrices, Vol. I, Chelsea Publishing Company, New York, 1959.

Department of Mathematics, University of Hong Kong, Hong Kong. 